\title{
Bone marrow mesenchymal stem cell implantation for the treatment of radioactivity-induced acute skin damage in rats
}

\author{
KAI ZHENG ${ }^{1}$, WEIZHEN WU ${ }^{1}$, SHUNLIANG YANG ${ }^{1}$, LIANGHU HUANG $^{1}$, JIN CHEN $^{1}$, \\ CHUNGUI GONG ${ }^{2}$, ZHICHAO FU ${ }^{2}$, LINLIN ZHANG ${ }^{1}$ and JIANMING TAN ${ }^{1}$ \\ ${ }^{1}$ Cell and Organ Transplant Institute; ${ }^{2}$ Radiotherapy Center, Fuzhou General Hospital, Fuzhou, Fujian 350025, P.R. China
}

Received September 26, 2014; Accepted June 22, 2015

DOI: $10.3892 / \mathrm{mmr} .2015 .4270$

\begin{abstract}
The present study aimed to observe the role of mesenchymal stem cells (MSCs) in the repair of acute skin damage caused by radiation. Rat bone marrow MSCs (BMSCs) were isolated and cultured in vitro. A rat model of radiation-induced acute skin damage was established by irradiation of the hind legs of Sprague-Dawley rats using a linear accelerator (45 Gy). After irradiation, rats were randomly divided into two groups: BMSC group and control group. Rats in the BMSC group were treated with a tail vein injection of $2 \times 10^{6}$ BMSCs $(1 \mathrm{ml})$ immediately after irradiation and a local multipoint injection of $2 \times 10^{6} \mathrm{BMSCs}$ at the injured area two weeks later. Then the wound healing of each rat was observed. The expression of transforming growth factor (TGF)- $\beta 1$, stromal cell-derived factor-1 (SDF-1) and prostaglandin E2 (PGE2) in the wounded tissues was determined by immunohistochemistry. The results demonstrated that skin damage was milder in the BMSC group than in the control group. Moreover, the speed of healing in the BMSC group was better than that in the control group. In addition, the wound score, it was significantly lower in the BMSC group than in the control group $(\mathrm{P}<0.05)$. The expression of PGE2 and TGF- $\beta 1$ in the BMSC group was also significantly lower than that in the control group $(\mathrm{P}<0.05)$, whereas the SDF-1 expression was significantly higher in the BMSC group than that in the control group $(\mathrm{P}<0.05)$. BMSCs can effectively reduce inflammation and fibrosis in the wounded skin and promote the repair of acute radioactive skin injury. Thus, may be developed as a novel treatment for wound healing.
\end{abstract}

\section{Introduction}

Nuclear technology has been widely used in a number of fields, including industry, agriculture, military and medicine.

Correspondence to: Dr Jianming Tan, Cell and Organ Transplant Institute, Fuzhou General Hospital, 156 Xi'erhuan North Road, Fuzhou, Fujian 350025, P.R. China

E-mail: kaizhengdoc@163.com

Key words: cell therapy, bone marrow mesenchymal stem cells, radioactive skin damage, rats
Since the Chernobyl nuclear accident (1) and Fukushima nuclear leakage, acute radiation injury has gained increased attention (2). Skin as a barrier coating on the surface of human body is easily damaged by radiation. In the process of clinical radiotherapy, $95 \%$ of the patients show a degree of skin damage (3). Radioactive skin damage usually takes a long time to heal, is characterized by recurrent episodes of necrotic ulcers, intense pain and potential cancer development in the long-term. It brings great physical and mental suffering to the patient. Thus, effective treatment methods are urgently required (4).

Bone mesenchymal stem cells (BMSCs) are a type of somatic stem cell with self-renewal and multi-directional differentiation potential. Owing to the progress in cell engineering and tissue engineering, the application of BMSCs has gained increasing attention (5). Studies have confirmed that BMSCs are effective in radioactive damage. François et al (6) found that human BMSCs infused into irradiated mice migrate towards the damaged skin. It was also verified that injection of BMSCs into rats with radioactively damaged skin improved the repair of the wounds (7). Lataillade et al (8) treated several patients with serious hip skin injuries caused by radiation with surgery and cell therapy, which obtained positive results. The mechanism underlying BMSCs promoting repair of radioactivity-induced damage remains unclear. It is reported that BMSCs differentiate into the cell type that has been damaged and thus promote the repair of wounds (9). However, another study demonstrated that BMSCs promote skin wound healing by paracrine mechanisms, such as the production of cytokines (10).

Transforming growth factor (TGF)- $\beta 1$, stromal cell-derived factor (SDF)-1 and prostaglandin E2 (PGE2) are important factors involved in inflammatory and anti-inflammatory responses (11-13). The aim of the present study was to observe the effects of BMSCs on the repair of radioactivity-induced acute skin injury in rats caused by a linear accelerator, and to investigate the underlying mechanism by detecting the expression of TGF- $\beta 1$, SDF-1 and PGE2.

\section{Materials and methods}

Preparation of rat BMSCs. Healthy male Sprague-Dawley rats ( $\mathrm{n}=43$; weight, 60-80 g) from Shanghai Laboratory Animal Center (Shanghai, China), were sacrificed by neck 
dislocation. Under aseptic conditions, bone marrow from the femur and tibia was collected by flushing the marrow cavity with physiological saline and was isolated using Ficoll separation solution (Hyclone, Logan, UT, USA). Bone marrow mononuclear cells prepared were then grown in complete Dulbecco's modified Eagle's medium (DMEM; HyClone Laboratories, Inc., Logan, UT, USA) with low glucose supplemented with $10 \%$ fetal bovine serum (FBS; Sigma-Aldrich, St. Louis, MO, USA), $100 \mathrm{U} / \mathrm{ml}$ penicillin (Sigma-Aldrich) and $100 \mathrm{U} / \mathrm{ml}$ streptomycin (Fuzhou Maixin Biotechnology Development Co., Ltd., Fuzhou, China) at $37^{\circ} \mathrm{C}$ in an atmosphere of $5 \% \mathrm{CO}_{2} / 95 \%$ air $/ 100 \%$ humidity. The medium was changed every three days. As the cells grew to $80-90 \%$ confluence, they were subcultured at a ratio of 1:3. This study was conducted in strict accordance with the recommendations in the Guide for the Care and Use of Laboratory Animals of the National Institutes of Health. The animal use protocol was reviewed and approved by the Institutional Animal Care and Use Committee (IACUC) of Fuzhou General Hospital (Fuzhou, China).

Osteogenic and adipogenic differentiation of rat BMSCs. Third passage BMSCs were seeded in 6-well plates at a density of $3 \times 10^{5}$ cells/well and were grown in osteoinduction medium (DMEM with high glucose supplemented with 10\% FBS, $10^{-7} \mathrm{M}$ dexamethasone, $10 \mathrm{mM} \beta$-sodium glycerophosphate and $50 \mathrm{mg} / 1$ vitamin $\mathrm{C}$ ) or adipoinduction medium (DMEM with high glucose supplemented with $10 \% \mathrm{FBS}, 10^{-6} \mathrm{M}$ dexamethasone, $0.5 \mathrm{mM}$ 3-isobutyl-1-methylxanthine, $10 \mu \mathrm{g} / \mathrm{ml}$ insulin and $0.2 \mathrm{mM}$ indomethacin) at $37^{\circ} \mathrm{C}$ in an atmosphere of $5 \% \mathrm{CO}_{2} / 95 \%$ air/ $100 \%$ humidity. The medium was changed every three days. Cells induced to osteogenesis were stained with Von Kossa stain three weeks later. Cells induced to adipogenesis were stained with Oil Red O (Sigma-Aldrich) two weeks later. The cells were observed under a Leica DVM6 optical microscope (Leica Microsystems GmbH, Wetzlar, Germany).

Identification of the surface antigens of rat BMSCs. Third passage BMSCs were harvested and adjusted to a concentration of $1 \times 10^{6} / \mathrm{ml}$. The cell suspension $(100 \mu \mathrm{l})$ was stained with PE-labeled CD29, CD34, CD45 and CD90 monoclonal antibodies (Biolegend, San Diego, CA, USA) at room temperature for $40 \mathrm{~min}$ in the dark, respectively. Then the cells were detected using a flow cytometer (EPICS XL, Beckman Coulter, Miami, FL, USA).

Establishment of the rat radioactivity-induced acute skin injury model. Sprague-Dawley rats $(\mathrm{n}=40)$ were anesthetized by intraperitoneal injection of $1 \%$ sodium pentobarbital. Then their right hip skin was irradiated using a linear accelerator (ZL00C-SN428, Varian Medical Systems, Inc., Palo Alto, CA, USA) with a total dose of $45 \mathrm{~Gy}(999 \mathrm{cGy} / \mathrm{min})$. The irradiation area was $2 \times 2 \mathrm{~cm}$. After irradiation, 40 rats were randomly divided into two groups: BMSC group and control group. Each rat in the BMSC group received tail vein injection of $2 \times 10^{6}$ BMSCs immediately after irradiation and local multipoint injection of $2 \times 10^{6}$ BMSCs around the damaged skin 2 weeks after irradiation. Rats in the control group received a tail vein injection of $1 \mathrm{ml}$ physiological saline.
Gross observation and wound score of damaged skin. After irradiation, the skin wounds of each rat were observed every day for a total of 8 weeks. The damaged skin was scored according to the acute radioactive skin reaction criteria of the International Union Against Cancer (14). Degree 0, the skin shows no change; degree I, the skin shows mild erythema, dry desquamation and reduced sweat; degree II, the skin shows obvious erythema, porphyritic moist dermatitis and moderate edema; degree III, the skin shows confluent moist dermatitis and pitting edema; degree IV, the skin shows necrosis, ulceration and bleeding.

Histopathologic examination of wounded skin. At 2, 4, 6 and 8 weeks after irradiation, the wounded skin was sampled from 3 rats of each group after the rats were anesthetized with $1 \%$ pentobarbital sodium by intraperitoneal injection. Then the skin tissues were fixed in $4 \%$ paraformaldehyde solution and embedded into paraffin. Thereafter, the tissues were cut into slices, mounted on slides and stained with hematoxylin and eosin or Masson's trichrome stain.

Determination of cytokine concentrations in the radioactively damaged skin by immunohistochemistry. At two, four, six and eight weeks after irradiation, the wounded skin tissue samples were obtained from three rats from each group under anesthesia by intraperitoneal injection of $0.4 \mathrm{ml} / 100 \mathrm{~g} 1 \%$ pentobarbital sodium. The tissues were sliced and mounted on 3-aminopropyltriethoxysilane-coated slides (Fuzhou Maixin Biotechnology Development Co., Ltd.). Following normal deparaffinization, enzyme closure with $3 \%$ hydrogen peroxide was performed, followed by antigen retrieval with citrate buffer (Sigma-Aldrich). The immunohistochemistry for PGE2, TGF- $\beta 1$ and SDF-1 was performed according to the manufacturer's instructions. The primary antibodies, including mouse anti-PGE2 monoclonal antibody (cat. no. mx18829; dilution, $1: 100)$, mouse anti-TGF- $\beta 1$ monoclonal antibody (cat. no. mx36721; dilution, 1:200) and mouse anti-SDF-1 monoclonal antibody (cat. no. mx22617; dilution, 1:200) were provided by Fuzhou Maixin Biotechnology Development Co., Ltd. The secondary antibody goat anti-mouse immunoglobulin G-horseradish peroxidase (cat. no. sc-2031; dilution 1:500) was provided by Santa Cruz Biotechnology, Inc. (Dallas, TX, USA).

Statistical analysis. All statistical processes were performed using SPSS 13.0 statistical software (SPSS, Inc., Chicago, IL, USA). Data are presented as the mean \pm standard deviation. Comparisons between two groups were conducted using a $\mathrm{t}$-test. $\mathrm{P}<0.05$ was considered to indicate a statistically significant difference.

\section{Results}

Morphology and differentiation. Microscopically, the primary rat BMSCs were round and of slightly different sizes. They were suspended in the medium with strong refractivity (Fig. 1A). After $24 \mathrm{~h}$, a number of the BMSCs adhered to the flask wall. After 2 or 3 days, the number of adherent cells increased rapidly and extended obvious pseudopodia. In addition, the cells became spindle-shaped. Within 3 to 4 days, 
Table I. Wound scores of radioactively damaged skin.

Time after radiation injury (weeks)

\begin{tabular}{lccccc}
\cline { 3 - 5 } Group & $\mathrm{n}$ & 2 & 4 & 6 & 8 \\
\hline Control & 20 & $2.6 \pm 0.5$ & $3.0 \pm 0.0$ & $2.3 \pm 0.5$ & $1.2 \pm 0.4$ \\
MSCs & 20 & $2.1 \pm 1.0$ & $2.4 \pm 0.7^{\mathrm{a}}$ & $1.5 \pm 0.5^{\mathrm{a}}$ & $0.3 \pm 0.5^{\mathrm{a}}$ \\
\hline
\end{tabular}

Data are presented as the mean \pm standard deviation. ${ }^{\mathrm{a}} \mathrm{P}<0.05$ vs. the control.
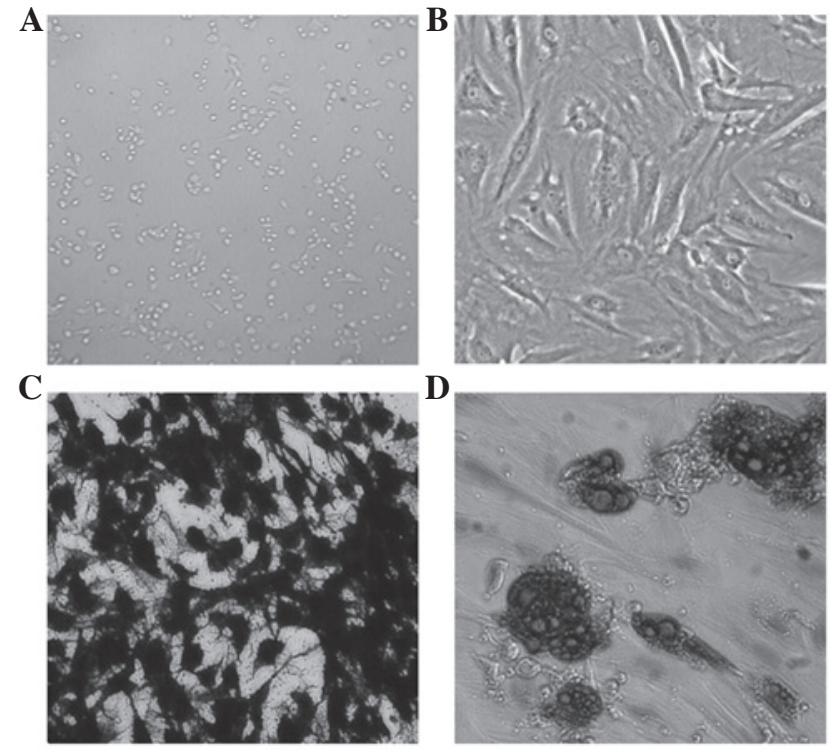

Figure 1. Morphology and differentiation of cultured rat BMSCs (magnification, x200). (A) Primary rat BMSCs suspended in medium, with strong refractivity. (B) Third passage rat BMSCs, had a uniform spindle shape, with abundant cytoplasm and clear nucleoli. (C) Osteo-induced BMSCs had calcium nodules demonstrated by Von Kossa staining. (D) Adipo-induced BMSCs presented with lipocytes as observed by Oil Red O staining. BMSCs, bone marrow mesenchymal stem cells.

A

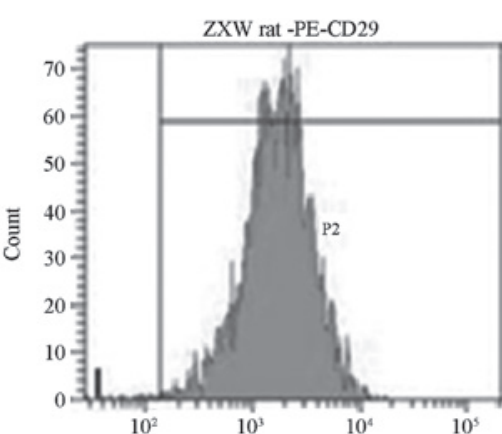

C

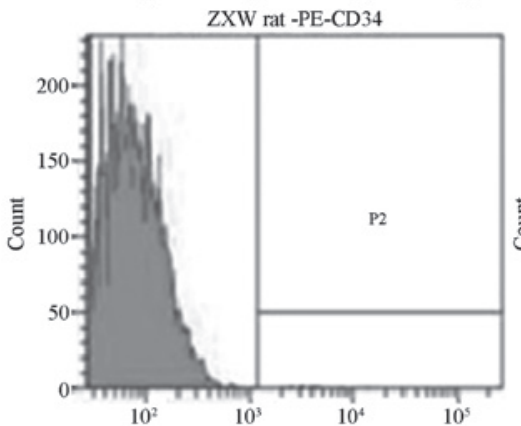

B
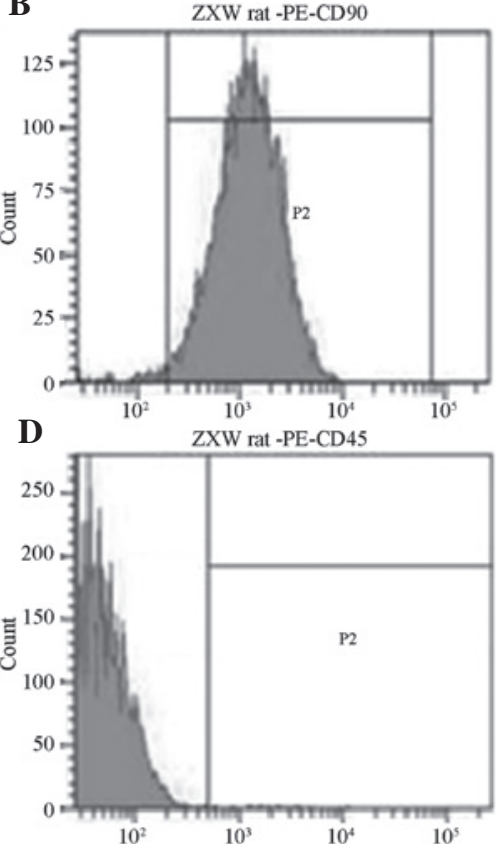

Figure 2. Surface markers of third passage rat BMSCs detected by flow cytometry. (A) CD29-positive and (B) CD90-positive cells accounted for 99.25 and $98.37 \%$ of the total cells, respectively. However, (C) CD34-positive and (D) CD45-positive cells only accounted for 1.12 and 1.03\%, respectively. BMSCs, bone marrow mesenchymal stem cells. 


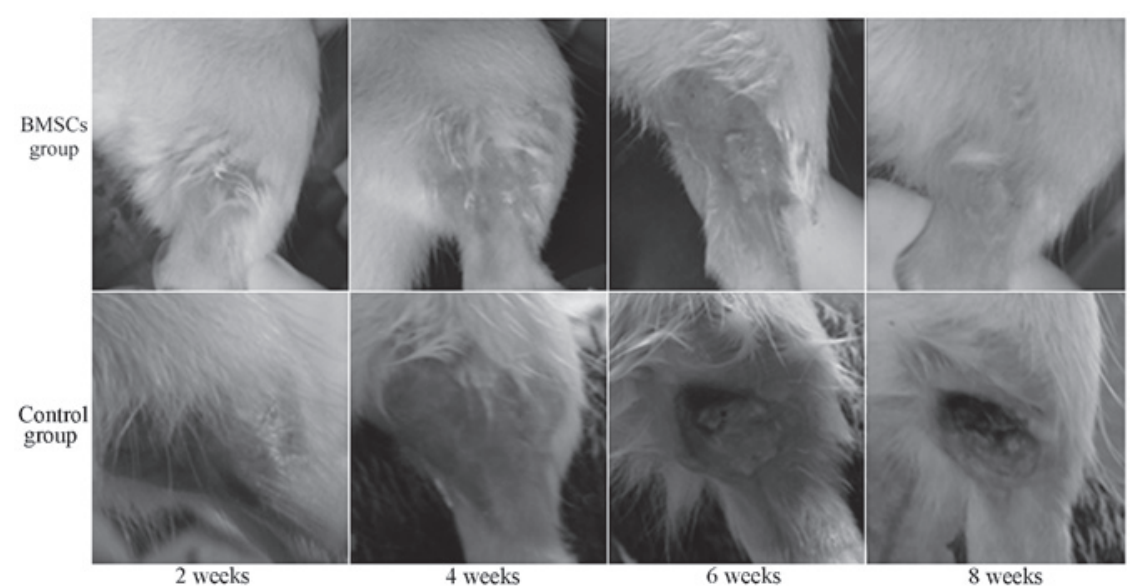

Figure 3. Wounded skin in rats after radiation exposure. In the BMSC group, the radioactive wound healed with time at a faster rate than the wounds in the rats form the control group.

A

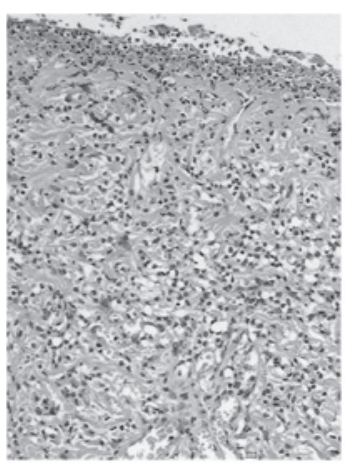

D

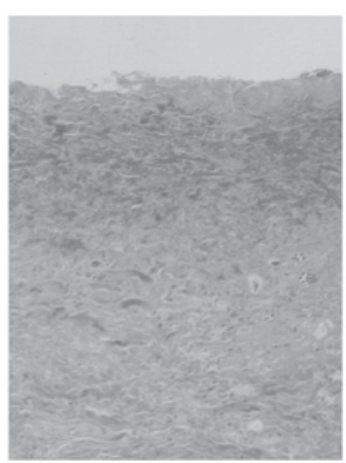

B

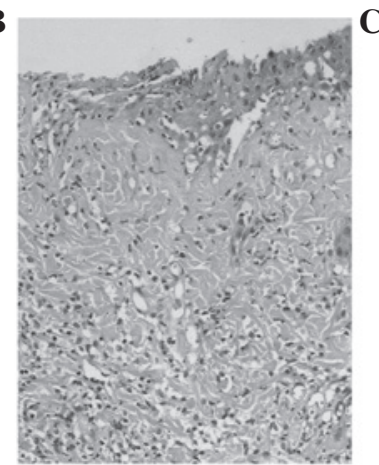

$\mathbf{E}$

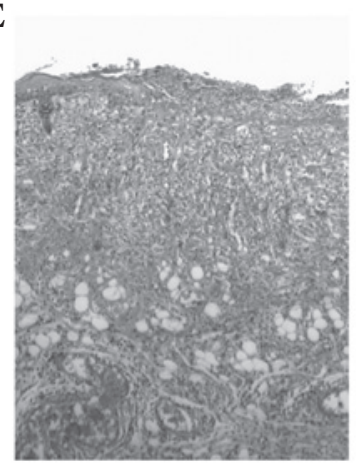

C

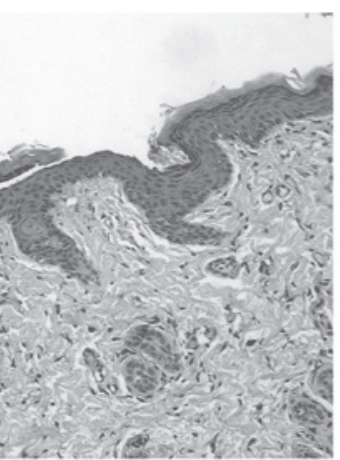

$\mathbf{F}$

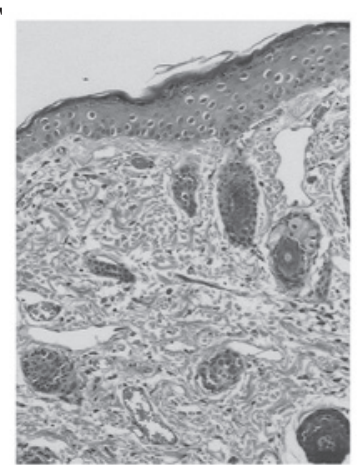

Figure 4. Histopathological examination of wound skin tissues in the BMSC group (H\&E staining and Masson's trichrome staining). Wounded skin 2 weeks after irradiation stained with $\mathrm{H} \& \mathrm{E}$ (A, x100) and Masson's trichrome staining (B, x100). H\&E staining showed a large number of infiltrated inflammatory cells and the disappearance of hair follicles and sebaceous glands; collagen fibers were stained blue by Masson's trichrome staining, which demonstrated collagen fiber degeneration, decomposition and fracture. Wounded skin at 6 weeks after irradiation was stained with H\&E (C, x100) and Masson's trichrome staining (D, x100). H\&E staining showed epidermis proliferation and migration towards the ulcerated area; subcutaneous fibroblasts migrated and aggregated and new granulation tissue could be seen. Masson's trichrome staining showed hyperplasia of collagen fibers and disordered arrangement of collagen. Wounded skin at 8 weeks after irradiation stained with H\&E (E, x100) and Masson's trichrome staining (F, x100). The wound had been completely covered with epithelium. Hair follicles and sebaceous glands were observed and collagen fibers arranged neatly. H\&E, hematoxylin and eosin.

cells grew in whirlpool colonies. Upon subculture to the third passage, the cells grew in clusters in relatively uniform, long fusiform shapes (Fig. 1B).

As the rat BMSCs grew in the osteoinduction medium, they gradually fused. With the extension of induction time, cells overlapped and the matrix gradually accumulated. In addition, mineral salt was deposited to form multiple nodules, which gradually merged. After 3 weeks of cultivation, the small-flake calcium nodules formed, which were stained black using
Von Kossa stain (Fig. 1C). BMSCs grown in adipoinduction medium presented with small lipid droplets in the cytoplasm after 1 week and the cells were arranged in a disorderly manner. After 2 weeks, highly refractive lipid droplets were observed in the cytoplasm, which could be stained with Oil Red O staining (Fig. 1D).

Surface markers. Third passage BMSCs showed positive expression of CD29 and CD90, but almost no expression of CD34 
A
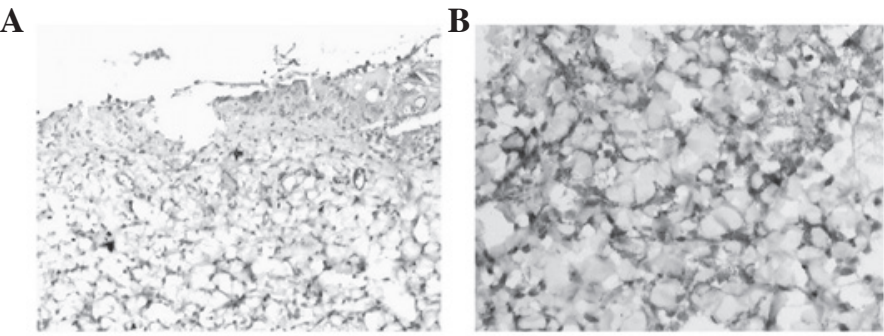

C
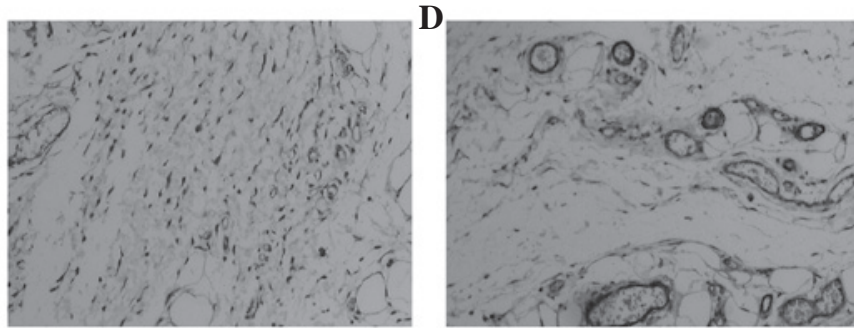

$\mathbf{E}$

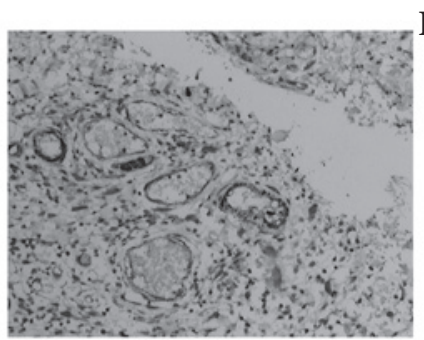

$\mathbf{F}$

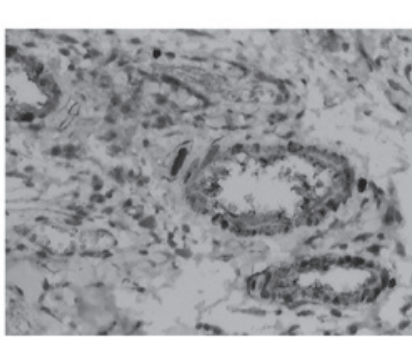

Figure 5. Expression of cytokines in wounded skin in the BMSC group (immunohistochemical staining, A, C and E, x100; B, D and F, x200). (A and B) PGE2 mainly expressed in fibroblasts and inflammatory cells; (C and D) TGF- $\beta 1$ mainly expressed in the hair follicle cells, vascular endothelial cells, fibroblasts and inflammatory cells; (E and F), SDF-1 was mainly distributed in new skin cells, fibroblasts and capillary endothelial cells. PGE2, prostaglandin E2; TGF- $\beta 1$, transforming growth factor- $\beta 1$; SDF-1, stromal cell-derived factor-1.

and CD45, as determined by flow cytometry. CD29-positive cells accounted for $99.25 \%$ and CD90-positive cells accounted for 98.37\%, while CD34-positive and CD45-positive cells only accounted for 1.12 and 1.03\%, respectively (Fig. 2).

Gross observation and the ratings of radioactively damaged skin. There were two fatalities in the control group following irradiation. The other rats presented with depilation, red swelling, mild erosion and slight seepage on the skin at 2 weeks after irradiation. At 4 weeks after irradiation, the wounded skin was erosive and formed into ulcers. After 6 weeks, the wounded skin began to dry and heal gradually, but the healing was slow. At 8 weeks after irradiation, new epithelium could be observed around the ulcer, but the wound healing was still incomplete (Fig. 3).

No rats in the BMSC group died following irradiation. At 4 weeks after irradiation, rats presented with exudation and erosion of the skin, but the depth and area of ulcers was significantly milder than that in the control group. At 6 weeks after irradiation, new epithelial growth could be observed at the injured site. After 8 weeks, the wound was almost healed and sparse hairs grew on the new skin (Fig. 3). At 4, 6 and 8 weeks after irradiation, the wound scores in the BMSC group were significantly lower than those in the control group, respectively $(\mathrm{P}<0.05$, Table I)

Histopathological results. The wounded skin in the control group revealed epidermal shedding and ulcer formation and a large number of inflammatory cells infiltrated 2 weeks after irradiation. The surface layer was made up of necrotic tissue, in which the hair follicle, sebaceous glands and other adnexa disappeared (Fig. 4A). Collagen fiber degeneration, decomposition and breakage could also be seen (Fig. 4B). At 6 weeks after irradiation, the proliferated epidermis gradually moved towards the ulcerated area. Combined with the migration and aggregation of subcutaneous fibroblasts, novel granulation tissue formed (Fig. 4C). Collagen fibers proliferated and arranged in a disordered manner (Fig. 4D). The degree of necrosis and the extent of inflammatory cell infiltration in the BMSC group was milder than that in the control group. New granulation tissue and epidermal hyperplasia could be observed 4 weeks after irradiation. Gradually, complete coverage of epithelium, production of hair follicles and sebaceous glands, a small degree of inflammatory cell infiltration and orderly arrangement of collagen fibers was observed 8 weeks after irradiation (Fig. 4E and F).

Expression of cytokines in the wounded skin. Prostaglandin E2 (PGE2) is important in inflammation. As detected by immunohistochemistry (IHC), PGE2 was predominantly expressed in fibroblasts and inflammatory cells (Fig. 5A and B). At 2 and 4 weeks after irradiation, the positive expression of PGE2 was prominently higher in the BMSC group than in the control group, respectively $(\mathrm{P}<0.05)$.

TGF- $\beta 1$ is involved in the inflammatory response at the early stages of damage and the tissue fibrosis process. The immunohistochemical staining of TGF- $\beta 1$ revealed that TGF- $\beta 1$ was predominantly distributed in the epithelium, hair 
follicle cells, basal vascular endothelial cells and fibroblasts around the ulcer (Fig. 5C and D). TGF- $\beta 1$ was expressed at 2, 4 and 6 weeks after irradiation and its expression in the BMSC group was significantly lower than its expression in the control group 4 and 6 weeks after irradiation, respectively $(\mathrm{P}<0.05)$.

SDF-1 is one of the predominant chemotactic factors in vivo, which contributes to the proliferation and migration of endothelial cells and accelerates neovascularization. The IHC results showed that SDF-1 is mainly expressed in the hair follicles, new skin cells, fibroblasts and capillary endothelial cells around the edge of the wounds (Fig. 5E and F). At 2, 4 and 6 weeks, the positive expression of SDF-1 in the MSCs group was markedly higher than its expression in the control group $(\mathrm{P}<0.05)$.

\section{Discussion}

MSCs are characterized by their potential for self-renewal and multi-directional differentiation, migrating towards damaged tissues to exert a reparative role and secrete a variety of growth factors. A large body of studies has shown that MSCs are involved in tissue repair in intestinal injury caused by radiation $(15,16)$, lung injury $(17,18)$, salivary gland damage (19) and combined radiation burn injury (20). In this study, radioactivity-induced acute skin damage was treated with BMSCs and it was demonstrated that BMSCs had protective effects. BMSCs not only reduced the extent of injury but also promoted repair by secreting cytokines, such as TGF- $\beta 1$, SDF-1 and PGE2.

It is reported that the reason that radioactive skin damage is difficult to heal is that the local vascular injury leads to microcirculation disturbance and tissue collagen fiber damage (21). Hu et al (22) reported that an appropriate quantity of MSCs promotes the repair of damage in hematopoiesis and immune organs, exhibiting a protective role in mice with acute radiation injury. Agay et al (23) established a minipigs model and found that the application of MSCs can significantly reduce the extent of radioactive injury and result in lymphocyte infiltration and angiogenesis in the dermis and subcutaneous tissue. In this study, the inflammatory reaction and the depth and area of ulcers in the MSCs group was significantly milder than that of the control group 2 weeks after irradiation, indicating that MSCs have a protective effect on radioactivity-induced skin damage.

Deng et al (24) treated C57BL/6 mice receiving a lethal dose of irradiation with fluorescence-labeled BMSCs and found that donor BMSCs migrated to the skin and converted into skin cells, so as to regenerate skin tissue. Moroz et al (25) also demonstrated that the implanted MSCs migrated to the damaged skin and subcutaneous injection of MSCs surrounding the damaged skin at 8 days after irradiation accelerated the healing of skin ulcers. It is also reported that treatment with MSCs can enhance the growth of hair in mice with radioactivity-induced injuries (26). The present results showed that BMSCs promoted the growth of granulation tissue and neovessels, as well as collagen hyperplasia at 6 weeks after irradiation. At 8 weeks after irradiation, epithelialization and increased cutaneous appendages were observed in the BMSC group, suggesting that BMSCs can accelerate wound healing, consistent with the above results.
TGF- $\beta 1$ is involved in the inflammatory response at the early stages of injury (27). It is also involved in fibrosis by promoting the transport of fibronectin and epithelial cells during the process of tissue-repair (11). SDF-1 is a chemokine involved in the process of stem cell homing (12). PGE is widely distributed in vivo, among which PGE2 exhibits an important regulatory role in physiological processes, such as inflammatory and anti-inflammatory responses (13). The results of the present study showed that BMSCs could prominently reduce the expression of TGF- $\beta 1$ and PGE2, and significantly increase SDF-1 expression in the irradiated rats as compared with the control group. It was hypothesized that BMSCs promote wound healing by inhibiting the expression of PGE2 and TGF- $\beta 1$, reducing local inflammatory reactions and inhibiting fibrosis at the inflammatory reaction stage. During wound repair, BMSCs can promote the production of SDF-1 by fibroblasts and thereby promote the migration of BMSCs to the injury site. These results were consistent with the results from Horton et al (28).

In conclusion, BMSCs effectively promote the repair of radiation-induced acute skin injury by secreting a variety of cytokines to communicate with the microenvironment of the wounded skin and lead to inhibition of the inflammatory response, increased chemotaxis, proliferation and prevent fibrosis. With the development of tissue engineering and cell engineering, BMSCs may become a novel method for the treatment of wounds caused by radioactivity. However, the appropriate BMSC treatment timing, treatment pathway and administration quantity requires further investigation in future studies.

\section{Acknowledgements}

The present study was supported by grants from the key project of the Army 'Twelfth Five-year' Science and Technology Plan (grant no. BWS11J004), the key project of the Science and Technology Plan of Nanjing Military Command (grant no. 10z031) and the Science and Technology Innovation Platform Construction Project of Fujian province, China.

\section{References}

1. Ogrodnik A, Hudon TW, Nadkarni PM and Chandawarkar RY: Radiation exposure and breast cancer: Lessons from Chernobyl. Conn Med 77: 227-234, 2013.

2. Heslet L, Bay C and Nepper-Christensen S: Acute radiation syndrome (ARS) treatment of the reduced host defense. Int J Gen Med 5: 105-115, 2012.

3. Porock D, Nikoletti S and Kristjanson L: Management of radiation skin reactions: Literature review and clinical application. Plast Surg Nurs 19: 185-192, 1999.

4. Kamiya H: Radiation-induced skin injuries. Nihon Rinsho 70: 427-430, 2012 (In Japanese).

5. Sharma RR, Pollock K, Hubel A and McKenna D: Mesenchymal stem or stromal cells: A review of clinical applications and manufacturing practices. Transfusion 54: 1418-1437, 2014.

6. François S, Bensidhoum M, Mouiseddine M, et al: Local irradiation not only induces homing of human mesenchymal stem cells at exposed sites but promote their widespread engraftment to multiple to organs: A study of their quantitative distribution after irradiation damage. Stem Cells 24: 1020-1029, 2006.

7. François S, Mouiseddine M, Mathieu N, Semont A, Monti P, Dudoignon N, Saché A, Boutarfa A, Thierry D, Gourmelon P and Chapel A: Human mesenchymal stem cells favour healing of the cutaneous radiation syndrome in a xenogenic transplant model. Ann Hematol 86: 1-8, 2007. 
8. Lataillade JJ, Doucet C, Bey E, Carsin H, Huet C, Clairand I, Bottollier-Depois JF, Chapel A, Ernou I, Gourven M, et al: New approach to radiation burn treatment by dosimetry-guided surgery combined with autologous mesenchymal stem cell therapy. Regen Med 2: 785-794, 2007.

9. Li H, Fu X, Ouyang Y, Cai C, Wang J and Sun T: Adult bone-marrow-derived mesenchymal stem cells contribute to wound healing of skin appendages. Cell Tissue Res 326: 725-736, 2006.

10. Galeano M, Altavilla D, Cucinotta D, Russo GT, Calò M, Bitto A, Marini H, Marini R, Adamo EB, Seminara P, et al: Recombinant human erythropoietin stimulates angiogenesis and wound healing in the genetically diabetic mouse. Diabetes 53: 2509-2517, 2004.

11. Rolfe KJ, Irvine LM, Grobbelaar AO and Linge C: Differential gene expressionin response to transforming growth factor-beta 1 by fetal and posnata1 dermal fibroblasts. Wound Repair Regen 15: 897-906, 2007.

12. Li Q, Zhang A, Tao C, Li $X$ and Jin $P$ : The role of SDF-1-CXCR4/CXCR7 axis in biological behaviors of adipose tissue-derived mesenchymal stem cells in vitro. Biochem Biophys Res Commun 441: 675-680, 2013.

13. Zhao X, Liu L, Liu D, Fan H, Wang Y,Hu Y and Hou Y: Progesterone enhances immunoregulatory activity of human mesenchymal stem cells via PGE2 and IL-6. Am J Reprod Immunol 68: 290-300, 2012.

14. Wei R, Jiang W, Su J,He L, Yang Z and Jiang W: Intensity modulated radiation therapy for 90 untreated nasopharyngeal carcinoma. Zhong Nan Da Xue Xue Bao Yi Xue Ban 37: 173-178, 2012 (In Chinese).

15. Chang P, Qu Y, Liu Y, Cui S, Zhu D, Wang H and Jin X: Multi-therapeutic effects of human adipose-derived mesenchymal stem cells on radiation-induced intestinal injury. Cell Death Dis 4: e685, 2013.

16. Linard C, Busson E, Holler V, Strup-Perrot C, Lacave-Lapalun JV, Lhomme B, Prat M, Devauchelle P, Sabourin JC, Simon JM, et al: Repeated autologous bone marrow-derived mesenchymal stem cell injections improve radiation-induced proctitis in pigs. Stem Cells Transl Med 2: 916-927, 2013.

17. Kuova LV, Konoplyannikov AG, Pasov VV, Ivanova IN, Poluektova MV and Konoplyannikova OA: Possibilities for the use of autologous mesenchymal stem cells in the therapy of radiation-induced lung injuries. Bull Exp Biol Med 147: 542-546, 2009.

18. Wang H, Yang YF, Zhao L, Xiao FJ, Zhang QW, Wen ML, Wu CT, Peng RY and Wang LS: Hepatocyte growth factor gene-modified mesenchymal stem cells reduce radiation-induced lung injury. Hum Gene Ther 24: 343-353, 2013.
19. Lim JY, Ra JC, Shin IS, Jang YH, An HY, Choi JS, Kim WC and Kim YM: Systemic transplantation of human adipose tissue-derived mesenchymal stem cells for the regeneration of irradiation-induced salivary gland damage. PLoS One 8: e71167, 2013.

20. Hao L, Wang J, Zou Z, Yan G, Dong S, Deng J, Ran X, Feng Y, Luo C, Wang Y and Cheng T: Transplantation of BMSCs expressing hPDGF-A/hBD2 promotes wound healing in rats with combined radiation-wound injury. Gene Ther 16: 34-42, 2009.

21. Millar WT, Van Den Aardweg GJ, Hopewell JW and Canney PA: Repair kinetics in pig epidermis: An analysis based on two separate rates of repair. Int J Radiat Biol 69: 123-140, 1996

22. Hu KX, Sun QY, Guo M and Ai HS: The radiation protection and therapy effects of mesenchymal stem cells in mice with acute radiation injury. British J Radiol 83: 52-58, 2010.

23. Agay D, Scherthan H, Forcheron F, Grenier N, Hérodin F, Meineke V and Drouet M: Multipotent mesenchymal stem cell grafting to treat cutaneous radiation syndrome: Development of a new minipig model. Exp Hematol 38: 945-956, 2010

24. Deng W, Han Q, Liao L, Li C, Ge W, Zhao Z, You S, Deng H, Murad F and Zhao RC: Engrafted bone marrow-derived flk- (1+) mesenchymal stem cells regenerate skin tissue. Tissue Eng 11: $110-119,2005$.

25. Moroz BB, Onizhshenko NA, Lebedev VG, Deshevoŭ IuB, Sidorovich GI, Lyrshchikova AV, Rasulov MF, Krasheninnikov ME and Sevast'ianov VI: The influence of multipotent mesenchymal stromal cells of bone marrow on process of local radiation injury in rats after local beta-irradiation. Radiats Biol Radioecol 49: 688-693, 2009 (In Russian).

26. Xie MW, Gorodetsky R, Micewicz ED, Mackenzie NC, Gaberman E, Levdansky L and McBride WH: Marrow-derived stromal cell delivery on fibrin microbeads can correct radiation-induced wound-healing deficits. J Invest Dermatol 133: 553-561, 2013.

27. Ueno T, Nakashima A, Doi S, Kawamoto T, Honda K, Yokoyama Y, Doi T, Higashi Y, Yorioka N, Kato Y, et al: Mesenchymal stem cells ameliorate experimental peritoneal fibrosis by suppressing inflammation and inhibiting TGF- $\beta 1$ signaling. Kidney Int 84: 297-307, 2013.

28. Horton JA, Hudak KE, Chung EJ, White AO, Scroggins BT, Burkeen JF and Citrin DE: Mesenchymal stem cells inhibit cutaneous radiation-induced fibrosis by suppressing chronic inflammation. Stem Cells 31: 2231-2241, 2013. 